\title{
A New Hybrid Model Based on Data Preprocessing and an Intelligent Optimization Algorithm for Electrical Power System Forecasting
}

\author{
Ping Jiang, ${ }^{1}$ Xuejiao Ma, ${ }^{1}$ and Feng Liu ${ }^{1,2}$ \\ ${ }^{1}$ School of Statistics, Dongbei University of Finance and Economics, Dalian, Liaoning 116025, China \\ ${ }^{2}$ School of Mathematics and Statistics, Lanzhou University, Lanzhou, Gansu 730000, China \\ Correspondence should be addressed to Xuejiao Ma; xuejiaomadufe@163.com
}

Received 20 May 2015; Accepted 23 August 2015

Academic Editor: Alkiviadis Paipetis

Copyright (C) 2015 Ping Jiang et al. This is an open access article distributed under the Creative Commons Attribution License, which permits unrestricted use, distribution, and reproduction in any medium, provided the original work is properly cited.

\begin{abstract}
The establishment of electrical power system cannot only benefit the reasonable distribution and management in energy resources, but also satisfy the increasing demand for electricity. The electrical power system construction is often a pivotal part in the national and regional economic development plan. This paper constructs a hybrid model, known as the E-MFA-BP model, that can forecast indices in the electrical power system, including wind speed, electrical load, and electricity price. Firstly, the ensemble empirical mode decomposition can be applied to eliminate the noise of original time series data. After data preprocessing, the back propagation neural network model is applied to carry out the forecasting. Owing to the instability of its structure, the modified firefly algorithm is employed to optimize the weight and threshold values of back propagation to obtain a hybrid model with higher forecasting quality. Three experiments are carried out to verify the effectiveness of the model. Through comparison with other traditional well-known forecasting models, and models optimized by other optimization algorithms, the experimental results demonstrate that the hybrid model has the best forecasting performance.
\end{abstract}

\section{Introduction}

The electrical power system is a type of production and consumption system that is composed of the power generation, transformation, transmission, and distribution of electricity and other aspects. With higher requirement for indices of electrical power system, it shows a tendency forward to the intelligent and optimal direction [1]. To build large-scale electrical power system with reasonable structure cannot only benefit the central management, unified schedule, and distribution of electrical power but also make for the development and utilization of regional energy resources and satisfy the increasing demand for electricity in each region to the largest extent. The appearance of electrical power system contributes to the initiation of the age of electricity, which makes the electrical energy with highly effective and pollution-free features applied widely. Nowadays, it has become one of the most important symbols for national economic development level. Therefore, to forecast related indices of electrical power system seems imperative and important. Wind speed, electrical load, and electricity price are chosen in this paper to carry out the forecasting of electrical power system.

As one of the most competitive new energies, wind energy is highly focused on by most countries in the world, including both developed and developing countries. In recent years, the development of wind power generation in Asia has made major achievements and the contribution of China has played a vital role [2]. On the one hand, wind energy in China is available and plentiful, especially in the northern region. On the other hand, the long coastline could provide plenty of offshore wind energy. Therefore, wind power generation on a large scale is feasible and can be applied. Accordingly, Chinese wind power industry is booming and promising. In addition to the current wind power bases, there are thousands of wind power bases that are in construction [3]. In view 
of the significance of wind energy, what is more important is the forecasting of wind speed. The short-term wind speed forecasting is meaningful in helping distribute power reasonably and ensure the quality of the power supply [4]. This paper focuses on the short-term wind speed forecasting.

Electrical load forecasting plays a pivotal role in the economic operation and the security of electrical power system, because several decisions that are significant to the economy are made based on the forecasting of electrical load, such as fuel allocation, short-term maintenance, and contingency planning load shedding. Thus, many countries all over the world seek effective methods to forecast the electrical load. China, with rapid rate of economic development as well as increasingly large demand for electricity, is supposed to pay more attention to the improvement of electrical load forecasting. Besides, although wind power generation has become more widely applied, the burning of coal still occupies the leading position in generating electricity, which would result in the emission of carbon dioxide that could bring enormous environmental damage [5]. So accurate and reliable electrical load forecasting is beneficial in planning the power generation and adjusting power policy and structures.

After forecasting the production and consumption of electricity, how to trade it, a very special commodity, is also a challenging issue for the electrical power system. Nowadays, the electricity is traded under market rules using derivative and spot contracts; however, the stability of trading price requires the constant balance between supply and demand. It is difficult to ensure the exact demanding for electricity, because many factors would have influence on the demand, including the intensity of business and everyday activities [7]. As for the supply of electricity, in the perspective of companies, the forecasting of electricity price has become a fundamental input to energy companies' decision-making mechanisms. Moreover, power portfolio managers show particular interest in the price forecasting from a few hours to a few months ahead so as to adjust their bidding strategy and decrease risk to maximize the profits.

Above all, it is meaningful to establish novel models to implement the forecasting of the electrical power system. The data of three chosen indices all belong to time series data with the features of nonlinearity and high fluctuation; therefore, it is not easy to get accurate and reliable forecasting results. Many researchers have made great efforts to develop the forecasting methods and two main approaches could be summarized, which are numerical simulation approach and statistical approach. Firstly, standard persistence methods are used as benchmarks to evaluate the new forecasting method. Generally, persistence is based on the assumption of no change in sky condition or constant clear sky index [8]. The persistence method works well when weather patterns change very little and features on the weather maps move very slowly. Nevertheless, if weather conditions change significantly from day to day, the persistence method usually breaks down and is not the best forecasting method to use. Compared with the numerical simulation approach, the statistical approach is applied more widely and is more popular, which mainly includes time series analysis, artificial neural network, and other related new hybrid models.
Firstly, in the aspect of time series analysis, it mainly deals with data ordered by time through identifying model, evaluating parameters, and building test and forecasting models. The most common time series analyses are autoregressive moving average (ARMA) model, autoregressive integrated moving average (ARIMA) model, and generalized autoregressive conditional heteroskedasticity (GARCH) model. Kavasseri and Seetharaman [9] applied fractional-ARIMA or f-ARIMA models to forecast the wind speed obtained from four potential wind generation sites in North Dakota on the day-ahead and two-day-ahead horizons. Compared with the persistence methods, the proposed model has great improvements in the performance of forecasting. Liu et al. [10] used ARMA-GARCH approach to model the mean and volatility of wind speed by applying the seven-year hourly data obtained from four different heights from an observation site in Colorado, USA. There were 10 models all together comprising the original form of five different GARCH approaches and their modified forms. They found out that the built model had a high forecasting accuracy and could catch the trend change of volatility and mean of wind speed effectively. In addition, they also proved that the ARMA-GARCH-M structures could improve the modeling sufficiency consistently due to the asymmetric time-varying and nonlinear features. Caporin and Preś [11] evaluated three approaches that could be used to forecast the wind speed intensity, including autoregressive Gamma (ARG) process, Gamma autoregressive (GAR) process, and ARFIMA-FIGARCH model. The first two models both combined autoregressive behaviors and Gamma distributions. In the model of ARG, the simulation of Gamma process went through the generation of random numbers from a twoparameter Gamma density, where parameters were timevarying. As for GAR, it was proposed in alternative conditional Gamma process based on three parameters' noncentral Gama density. Bouzerdoum et al. [12] combined two famous methods, which were the seasonal autoregressive integrated moving average method and the support vector machines (SVM) to forecast the short-term power and comparative study was introduced showing that the developed hybrid model performed better than both the single models. $\mathrm{Xu}$ et al. [13] established a new model with improved grey model (GM) and ARMA based on HP filter to forecast the final energy consumption. Through comparing with traditional statistical approaches, the experimental results demonstrated that the improved GM-ARMA model had excellent accuracy and higher level of reliability.

Secondly, as for the artificial neural network (ANN) method, it could be applied to address complicated nonlinear problems, because it has a strong ability of self-learning and self-adaptation. ANN is more likely used by combining with other models and methods. Cadenas and Rivera [14] developed ARIMA-ANN model to carry out the forecasting by using average hourly wind speed data obtained directly from the measurements at different sites for about one month. ARIMA was applied first to do the forecasting of the time series, and then ANN was used to take the nonlinear tendencies into consideration based on the obtained errors. The built hybrid model could forecast wind speed with a higher 
accuracy than ARIMA or ANN models separately according to the calculation of the mean error (ME), the mean square error (MSE), and the mean absolute error (MAE). Nan et al. [15] established a forecasting model composed of time series and back propagation neural network (BPNN) prediction model. As for the time series analysis, bias correction method on empirical orthogonal function (EOF) was proposed. The application of EOF was aimed at decomposing forecasting error and the proposed model EOF-BPNN could forecast the short-term wind speed with a higher accuracy.

Rahmani et al. [16] developed a new hybrid swarm technique (HAP) to forecast the energy output in a real wind farm located in Binaloud, Iran. HAP was optimized by two optimization algorithms that were two metaheuristic techniques, including ant colony optimization (ACO) and particle swarm optimization (PSO). The combination of two algorithms can both improve the forecasting accuracy and accelerate the convergence rate. The results indicated that the proposed hybrid model could estimate the output wind power based on the ambient temperature and wind speed with a mean absolute percentage error (MAPE) of 3.5\%. Geng et al. [17] introduced robust support vector regression (RSVR) and clonal selection principle of artificial immune algorithm combined with particle swarm optimization (CSAPSO) to get more accurate forecasting results. RSVR was applied to carry out the forecasting, and then CSAPSO was used to optimize the parameters in RSVR in order to improve the forecasting accuracy and save time. The established model performed better than six other competing models used in the study.

In order to reduce error and improve the forecasting accuracy, the noise reduction of input data has attracted larger and larger attention. Liu et al. [18] applied two hybrid forecasting models, including the Wavelet-particle swarm optimization- (PSO-) multilayer perceptron (MLP) and the Wavelet-genetic algorithm- (GA-) multilayer perceptron (MLP). The analysis results showed that both of the two proposed models were suitable for the diverse accuracy requirement in wind speed predictions and the contribution of Wavelet component was statistically significant. Zhang et al. [19] proposed four approaches that had better forecasting performance than traditional forecasting methods. The first two approaches applied PSO to optimize the parameters in the first-order and second-order adaptive coefficient (FAC and SAC) methods, and then the last two approaches employed the decomposition of the original data into seasonal and trend components. The application of decomposition of data to seasonal and trend components helped arrive at a higher forecasting accuracy through calculating the MSE, MAE, and the relative errors. Zhang el al. [20] applied WTT-SAM-RBFNN to forecast the wind speed. WTT meant Wavelet transform technique and it played a vital role in reducing the forecasting errors. SAM indicated seasonal adjustment method, which was also important in improving the forecasting accuracy. RBFNN was radial basis function neural network that was applied to carry out the forecasting. The use of two adjustments of original wind speed data made the forecasting accuracy improve to a great extent compared with the traditional forecasting methods.
The time series data of electrical power system is of high randomness, because it is affected by many factors, such as temperature, altitude, air pressure, and other related factors. Empirical mode decomposition (EMD) is applicable in eliminating the noise in the original series through carrying out the adaptive time-frequency decomposition based on the features of the time variation of signal [21]. However, the mode mixing problem resulting from the intermittency signal in the sifting process of EMD would affect the forecasting accuracy. Thus, ensemble empirical mode decomposition (EEMD) (see Algorithm 1) is introduced to surpass the defects of EMD. The white noise is added into the original signal so that it could have the decomposition scale of a uniform distribution based on the statistical feature of Gaussian white noise. EEMD could smooth the impulse interference effectively and address the mode mixing problem.

BPNN is a common forecasting method for its unique advantages, including nonlinear mapping capability, selflearning and self-adaptive ability, generalization ability, and fault-tolerant capacity. However, the structure of the BPNN network is uncertain, which could cause the high forecasting errors [22]. Therefore, this paper utilizes modified firefly algorithm (MFA) (see Algorithm 2) to optimize the weight and threshold values of the network. FA is a random and parallel optimization algorithm with low requirements for the function to be optimized, simple process, and fast convergent rate [23]. However, the largest defect of FA is that it is easy to trap into the local optimum, so this paper improves the FA in order to make it jump out of the local optimum and the hybrid model could improve the forecasting accuracy through combining with EEMD.

Our Contribution. Many scholars have made great efforts to seek out the methods to forecast the time series data, and this is because it is really a meaningful and challenging work, especially for the forecasting of wind speed, electrical load, and electricity price. Based on the reasons above, this paper tries to propose a new hybrid model, known as the E-MFA-BP, to be applied to the forecasting of electrical power system. Initially, the ensemble empirical mode decomposition (EEMD) is used to reduce the noise in the original data series and the effect of noise reduction is satisfactory. Then, after preprocessing the original data, the smoother data is used to carry out the forecasting. MFA is utilized to optimize the weight and threshold values of the network of BPNN in order to reduce the forecasting errors. Their combination successfully takes the advantage of each single algorithm. In general, our contribution could be summarized as one that proposes a hybrid model that is effective in the forecasting in the electrical power system.

The whole structure of this paper is given below. Firstly, Section 1 is the introduction containing the description of background of electrical power system and related researches on this field. Then, Section 2 introduces the components of the hybrid model, including ensemble empirical mode decomposition, modified firefly algorithm, back propagation neural network, and the essence of hybrid model proposed in this paper. Next, the experimental simulation and the evaluation of the hybrid model are included in Section 3. 


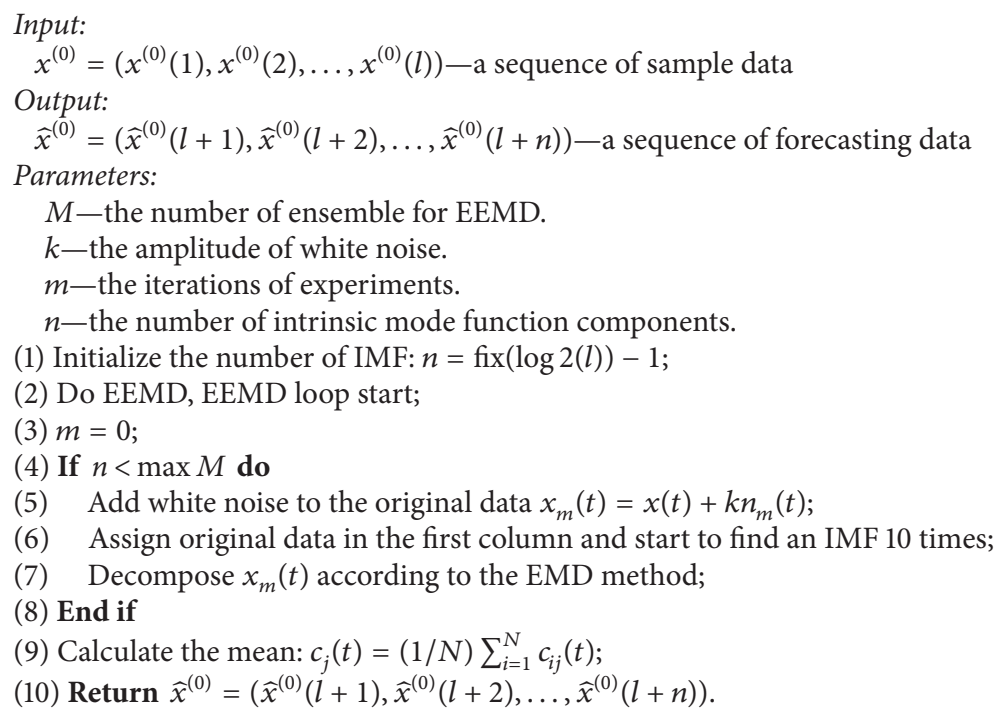

Algorithm 1: Pseudocode of EEMD.

\section{Input:}

The initial random value of weight $\alpha$ and threshold $b$ of BP artificial neural network. Output:

The global optimal extreme point $m_{\text {best }}$ and the best individual value $f_{\min }=\min \left(f\left(m_{\text {best }}\right)\right)$

\section{Parameters:}

$\mathrm{N}$ - the number of population of the particle swarm.

$c_{1}, c_{2}$-the cognitive and social weight of PSO algorithm. In usual, $c_{1}=c_{2}=2$.

$M$-the maximum iteration.

(1) Initialize the population $m_{i}(i=1,2, \ldots, n)$;

(2) $f\left(m_{i}\right)=$ update the light intensity after evaluating new solution;

(3) $t=0$;

(4) While $t<\max T$ do

(5) for $i=1: n$ all $n$ fireflies do

(6) for $j=1: i$ all $n$ fireflies do

(7) if $I_{j}>I_{i}$ then

(8) Move firefly $i$ towards $j$ using uniform distribution;

(9) end if

(10) end for

(11) Evaluate new solutions and update light intensity;

$$
\begin{aligned}
& x_{i}(t+1)=x_{i}(t)+(1-2 r) * R_{d}^{i}(t) * s_{-} \text {init } \\
& \bar{d}_{i}=\frac{1}{\left|N_{i}(t)\right|} \sum_{j \in N_{i}(t)}\left(\left\|x_{i}-x_{j}\right\|\right) ; \quad s_{i}(t)=s_{i}(t-1) * q
\end{aligned}
$$

(12) end for

(13) Rank fireflies and find the best individual;

(14) end while

Algorithm 2: Pseudocode of MFA.

The wind speed data of Shandong Peninsula in China and electrical load data and electricity price data from New South Wales (NSW) are used to test the performance of the model. Section 4 is the discussion and Section 5 includes conclusion and further work that summarize the ideas in the whole paper.

\section{Methods}

In this section, ensemble empirical mode decomposition, the modified firefly algorithm, and back propagation neural network are described. Next, the hybrid model E-MFA-BP proposed in this paper is introduced in detail. 
2.1. Ensemble Empirical Mode Decomposition (EEMD). To eliminate the mode mixing problem in the classical EMD method, ensemble empirical mode decomposition (EEMD) is proposed [24]. The core idea of EEMD is to make use of the statistical property of white noise; namely, its frequency would distribute uniformly. When Gaussian white noise is added to the signal, the signal should have continuity in different scales, and the feature of multiple extreme points would be changed as well [24]. The two important parameters are the value of amplitude $k$ and total number of EMD decompositions $M . k$ indicates the proportion of white noise in the standard deviation of the amplitude of the original signal [24]. To date, there has been no equation that could be used to calculate $M$ or $k$; therefore, based on the references, $M$ is 100 and $k$ is between 0.05 and 0.5 in this paper. The detailed steps of EEMD are described as follows [25].

Step 1. Initialize the number of ensemble $M$, the amplitude of white noise $k$, and iterations of experiments $m$, with $m=1$.

Step 2. Perform $m$ th experiments; the detailed steps are listed below:

(a) Add a random Gaussian white noise sequence to the original signal to obtain a new sequence with noise as

$$
x_{m}(t)=x(t)+k n_{m}(t),
$$

where $x(t)$ is the original signal that needs to be processed and $n_{m}(t)$ represents the random Gaussian white noise sequence.

(b) Decompose $x_{m}(t)$ according to the EMD method and obtain $n$ IMF components and one remaining component:

$$
\begin{aligned}
c_{i \cdot m}(t) & =h_{j \cdot m}(t), \quad i=1,2, \ldots, n, \\
r_{n \cdot m}(t) & =r_{n-1 \cdot m}(t)-c_{n \cdot m}(t),
\end{aligned}
$$

where $c_{i \cdot m}(t)$ represents $n$ components of the IMF and $r_{n \cdot m}(t)$ indicates the one remaining component.

(c) If $m<M, m=m+1$, return to Step 2; otherwise perform Step 3.

Step 3. Calculate mean of all components of the IMFs and remaining components in $m$ th EMD. Consider

$$
\begin{aligned}
& \overline{c_{i}}(t)=\sum_{m=1}^{M} \frac{c_{i \cdot m}(t)}{M}, \quad i=1,2, \ldots, n, \\
& \overline{r_{n}}(t)=\sum_{m=1}^{M} \frac{r_{n \cdot m}(t)}{M} .
\end{aligned}
$$

Step 4. Export $\overline{c_{i}}(t)(i=1,2, \ldots, n)$ and $\overline{r_{n}}(t)$, respectively, as $n$ IMF components and the one remaining component of EEMD.

\subsection{Firefly Algorithm}

2.2.1. Original Firefly Algorithm. The firefly algorithm (FA) is formed based on the imitation of the luminescence of fireflies in the natural world. In FA, the reasons for fireflies to attract each other depend on two major factors: brightness and attractiveness [26]. Firstly, the brightness of fireflies depends on their location, and the higher the value of the brightness, the better the place. Secondly, the attractiveness is also related to the brightness. Fireflies with higher brightness own higher attractiveness [27]. If each firefly has the same brightness, then they would move randomly. Both the brightness and the attractiveness are inversely proportional to the distance, which indicates that the brightness and attractiveness would decrease with the increase of the distance between each firefly [28]. Before applying FA, we define several terms.

Definition 1. Let $J\left(x_{i}(t)\right)$ be the objective function value of each firefly $i$ at the position $x_{i}(t)$ in $t$ th iteration. $l_{i}(t)$ represents the brightness of the current firefly and $\gamma$ denotes the update rate of brightness. So the update of brightness is defined as

$$
l_{i}(t)=(1-\rho) l_{i}(t-1)+\gamma J\left(l_{i}(t)\right) .
$$

Definition 2. Let the neighbor set $N_{i}(t)$ be $\left\{j: d_{i j}(t)<\right.$ $\left.r_{d}^{i}(t) ; l_{i}(t)<l_{j}(t)\right\}, 0<r_{d}^{i}(t) \leq r_{s}$, where $r_{s}$ is the perceived radius, so one defines the probability of individual $j$ moving to the neighbor set as

$$
p_{i j}(t)=\sum_{k \in N_{i}(t)}^{l_{j}(t)-l_{i}(t)}\left(l_{k}(t)-l_{i}(t)\right) .
$$

Definition 3. In the firefly algorithm, to update the location is important. So one defines the update of location as

$$
x_{i}(t+1)=x_{i}(t)+s\left(\frac{x_{j}-x_{i}}{\left\|x_{j}-x_{i}\right\|}\right),
$$

where $s$ means moving step length.

Definition 4. One defines the updated radius of dynamic decision domain:

$$
r_{d}^{i}(t+1)=\min \left\{r_{s}, \max \left\{0, r_{d}^{i}(t)+\beta\left(n_{i}-N_{i}(t)\right)\right\}\right\} .
$$

The detailed steps of FA are as follows [28].

Step 1. Initialize the basic parameters of FA. Population of fireflies is $m$, the largest attractiveness is $\beta_{0}$, and $\gamma$ is the light absorption coefficient. Maximum iteration is $\max T$ and searching precision is $\varepsilon$.

Step 2. Randomly initialize the location of fireflies and calculate the objective function value as the largest brightness $l_{0}$.

Step 3. Calculate the light intensity of fireflies and the attractiveness. The movement direction is decided by the light intensity:

$$
\begin{aligned}
& l=l_{0} \times e^{\gamma r_{i j},} \\
& \beta=\beta_{0} \times e^{\gamma r_{i j}^{2}},
\end{aligned}
$$

where $r_{i j}$ is the distance between firefly $i$ and firefly $j$. 
Step 4. Update the space location of fireflies and disturb the fireflies in the optimal location randomly:

$$
x_{i}=x_{i}+\beta_{0} e^{-\gamma r^{2}}\left(x_{j}-x_{i}\right)+\alpha(\text { rand }-0.5),
$$

where $x_{i}$ and $x_{j}$ are, respectively, the location of firefly $i$ and firefly j. $\alpha$ is the step size factor and is a constant between 0 and 1 . rand is a uniformly distributed generated number in $[0,1]$.

Step 5. Recalculate the brightness of fireflies according to the updated location.

Step 6. When searching precision is satisfied or the maximum iteration is arrived at, perform Step 7, or when $T=T+1$, return to Step 3.

Step 7. Export the global optimal extreme point and the best individual value.

2.2.2. Modified Firefly Algorithm (MFA). As demonstrated in [29], the computational performance of the FA is better in comparison with other optimization algorithms, such as GA and PSO [30]. The algorithm is simple with few parameters to be set. However, it still has some drawbacks, such as the low peak discovery rate, slow rate of convergence, and low precision of solution. In FA, each firefly has different searching scale, which is determined by the perceived radius. The movement of individual firefly depends on the excellent fireflies in the perceived radius; thus if there is no excellent firefly the individual location would not move. The reason for FA to search for the optimal solution locally and globally is determined by the movement of individual firefly towards excellent fireflies until finding the optimal solution. Such searching strategy cannot guarantee the individual firefly to give full play to the search, because the degree of dependency on surrounding potential information is too high, which would reduce the rate of convergence and discover rate of peak. On the other hand, with the increase of iteration, fireflies gather near the peak and tend to convergence and there is a very tiny distance between individuals and the peak [31]. However, if the step length is larger than the distance between individual firefly and the peak, the firefly would move to the other side of the peak. The optimal solution is still not obtained. If the peak cannot be found accurately, the algorithm would continue finding the peak in order to obtain the optimal solution, which would have an influence on the convergence rate [31].

Based on the discussion above, there are two main changes for the shortcomings of FA. The first one is called automatic random search and it is defined as follows.

Definition 5. If the individual firefly $i$ does not find a better individual, namely, the neighborhood set is empty, then the individual firefly $i$ would move and update location according to (10). If the location after moving is better than the location before moving, the former location would be saved, or the location stays unchanged. $r$ is the $N$-dimension random vector. Consider

$$
x_{i}(t+1)=x_{i}(t)+(1-2 r) * R_{d}^{i}(t) * s_{-} \text {init. }
$$

The second modification for FA is called adaptive step and we define it as follows.

Definition 6. Calculate the average distance among all individuals in the searching region according to (11) if the average distance is smaller than the step length $s$. Therefore, the updated step length is defined as

$$
\begin{aligned}
\bar{d}_{i} & =\frac{1}{\left|N_{i}(t)\right|} \sum_{j \in N_{i}(t)}\left(\left\|x_{i}-x_{j}\right\|\right), \\
s_{i}(t) & =s_{i}(t-1) * q .
\end{aligned}
$$

The first improvement of FA is to choose the movement direction randomly centered on the individual firefly when the individual firefly $i$ does not find the excellent individuals in the perceived range. To move one step towards the direction selected randomly could increase the possibility to find the local optimal solution in order to guide fireflies to move towards other individuals aimed at improving the rate of convergence. The second improvement is to add a distinguishing condition, according to which the step length decreases when the average distance between individual $i$ and all the other individuals in the neighborhood set is smaller than the fixed step length so as to weaken the shock around the optimal solution [31]. The reason for adding the condition is that the distance between individual and local optimal solution is very small with the increase of iterations.

The following list shows some important parameters in MFA that could have influence on the forecasting accuracy. The key parameters in MFA are listed as follows.

\section{Parameters in MFA}

$n$ : total number of the fireflies.

$r_{d}^{i}$ : decision radius of firefly $i$.

Iter_max: the maximum iteration.

$l_{i}$ : brightness of firefly $i$.

$r_{0}$ : initial perceived radius.

$d_{i j}$ : euclidean distance between firefly $i$ and firefly $j$.

$r_{s}^{i}$ : perceived radius of firefly $i$.

$s$ : moving step length.

$\rho_{i j}$ : moving probability of firefly $i$ towards firefly $j$.

$p$ : attenuation coefficient of brightness.

It is important to set reasonable parameters in MFA so as to find out the best performance in MFA; however, there are no unified methods on how to set parameters. According to [31], we know that the moving step length $s$ is the most important factor that affects the algorithm performance; then the second key factor is the population size $n$ of the firefly. 
The perceived radius $r_{s}^{i}$ ranks to the third place. When the moving step length $s$ is too small, the forecasting errors would become smaller; however, the rate of convergence nearly stops. Accordingly, the suitable moving step length would lead all the fireflies to gather around the objective source, which results in better forecasting performance.

2.3. Back Propagation Neural Network. Back propagation artificial neural network is a feed-forward neural network practiced by back propagation algorithm, and it is one of the neural network modes applied widely $[32,33]$. It adopts the error back propagation algorithm to learn and train the neural network, and this algorithm is on the basis of the error function gradient of the network. The three-layer network structure of BPNN is the most common applied to the forecasting and how to ensure the number of node of each layer is the key for the network. After ensuring the number of nodes, the parameters of BPNN need to be optimized, including weight and threshold, in order to reduce errors. The adaptability of BPNN is achieved through continuously adjusting the weight and threshold.

Step 1. Initialize the network. The node number of input layer, hidden layer and output layer can be determined by the input and output sequence $(X, Y)$. Initialize weight $w_{i j}$ between the input layer and hidden layer and weight $w_{j k}$ between the hidden layer and output layer. Initialize the threshold value of hidden layer $a$ and threshold value of output layer $b$. Set the learning rate $\eta$ and neuron activation function $f$. Consider

$$
f(x)=\frac{1}{1+e^{-x}} .
$$

Step 2. Calculate output of hidden layer $H$ based on $X, w_{i j}$, and $a$. Consider

$$
H_{j}=f\left(\sum_{i=1}^{n} w_{i j} x_{i}-a_{j}\right), \quad j=1,2, \ldots, l,
$$

where $l$ is the node number of the hidden layer and $f$ is the activation function of the hidden layer.

Step 3. Calculate output of the output layer according to $H$, $w_{j k}$, and $B$. Consider

$$
O_{k}=\sum_{j=1}^{l} H_{j}\left(w_{j k}-b_{k}\right) \quad k=1,2, \ldots, m .
$$

Step 4. Calculate error e. Consider

$$
e_{k}=Y_{k}-O_{k} \quad k=1,2, \ldots, m .
$$

Step 5. Update weights $w_{i j}$ and $w_{j k}$. Consider

$$
\begin{gathered}
w_{i j}=w_{i j}+\eta H_{j}\left(1-H_{j}\right) x(i) \sum_{k=1}^{m} w_{j k} e_{k} \\
i=1,2, \ldots, n ; j=1,2, \ldots, l, \\
w_{j k}=w_{j k}+\eta H_{j} e_{k} \quad j=1,2, \ldots, l ; k=1,2, \ldots, m .
\end{gathered}
$$

Step 6. Update the threshold values $a_{j}$ and $b_{k}$. Consider

$$
\begin{aligned}
& a_{j}=a_{j}+\eta H_{j}\left(1-H_{j}\right) \sum_{k=1}^{m} w_{j k} e_{k} \quad j=1,2, \ldots, l, \\
& b_{k}=b_{k}+e_{k}, \quad k=1,2, \ldots, m .
\end{aligned}
$$

Step 7. Judge whether iteration is in the end, or return to Step 2.

2.4. Hybrid E-MFA-BP Model. BPNN is a commonly used method to carry out forecasting; however, it still has some limitations, such as the determination of weight and threshold value, which could have a large influence on the accuracy of forecasting [34]. Therefore, this paper applies MFA to optimize the weight and threshold values of BPNN to reduce the forecasting errors. In addition, for the sake of improving the forecasting accuracy to a greater degree, this paper applies EEMD to address the original data through decomposing the noise. In summary, the basic idea of E-MFA-BP is to use EEMD to decompose the noise in the original data firstly. Then, the network structure of BPNN would be ensured based on the input and output parameters. Each individual in the population contains all the weight and threshold values of BPNN; therefore, the best individual could be found out through calculating the fitness value and using the fitness function and updating location, as well as the radius of decision. Moreover, a value must be assigned to the original weight and threshold of BPNN based on the best individual obtained from MFA. After optimizing BPNN, the globally optimal solution could be obtained. Figure 1 shows the whole forecasting process of the hybrid model, and the detailed steps of E-MFA-BP are described as follows.

Step 1. EEMD method is initially applied to preprocess the original data.

Step 2. BPNN is employed to carry out the forecasting by using the data processed by the EEMD method.

Step 3. MFA is introduced to optimize the weight and threshold values of BPNN.

Step 4. The absolute percentage error (APE), mean absolute error (MAE), mean square error (MSE), mean absolute percentage error (MAPE), and maximum percentage error (MPE) are used to evaluate the forecasting performance of the proposed hybrid model.

\section{Experimental Simulation and Results Analysis}

The performance of the hybrid model proposed in this paper is tested by three experiments, including wind speed, electrical load, and electricity price data sets collected from the Shandong Peninsula in China and NSW. Five indices, including APE, MAPE, MSE, MAE, and MAP, are used to evaluate the forecasting quality of the E-MFA-BP model. 


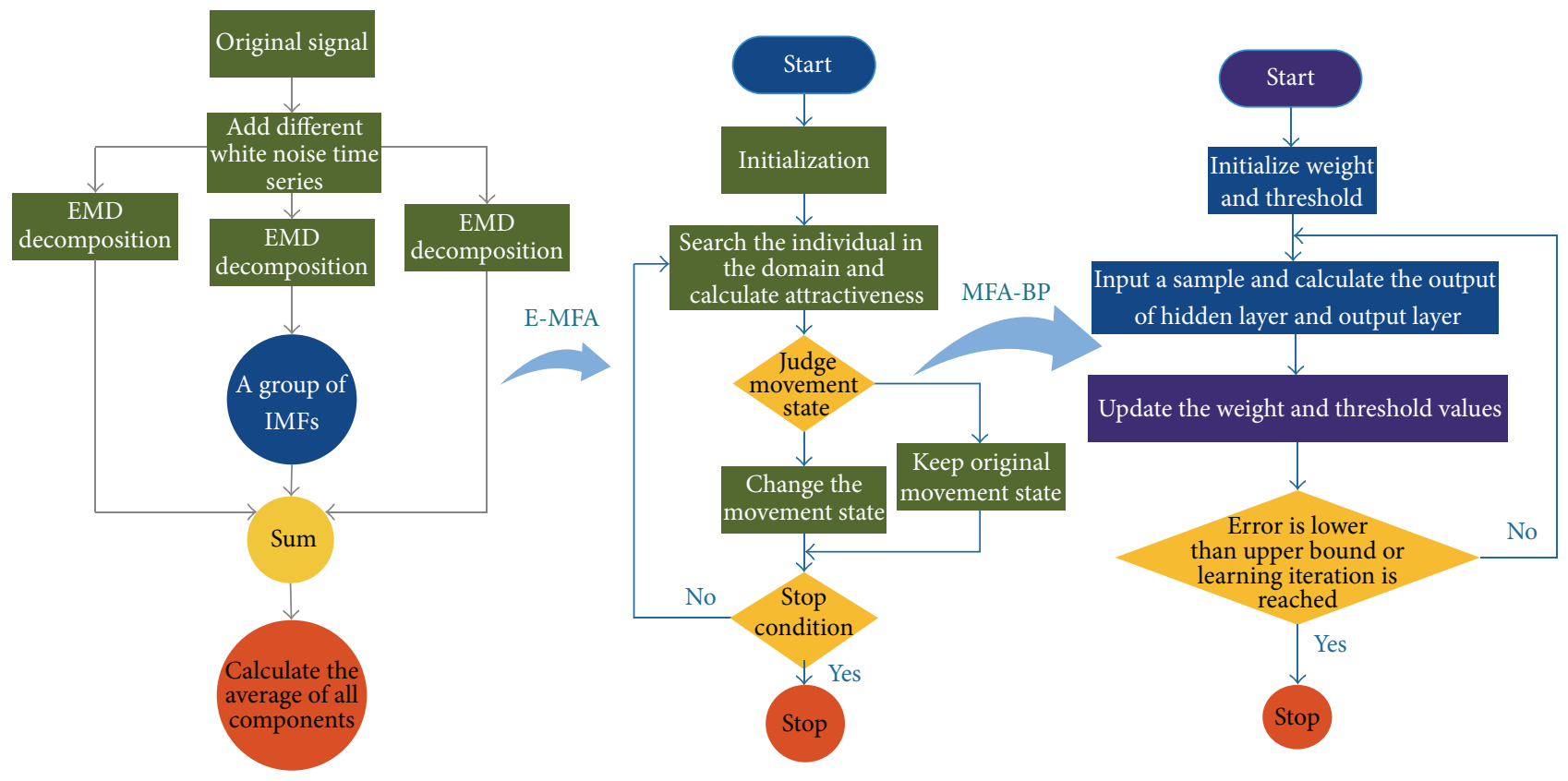

FIgURE 1: Forecasting process of the hybrid model E-MFA-BP.

3.1. Data Sets. Short-term wind speed data set is collected from Shandong Peninsula of China covering the scale from June 1 to June 10 in three observation sites. The description of short-term wind speed time series data is shown in Figure 2. The training data set is the data of the first five days and the testing data set is the data in the sixth day. For example, data from June 1 to June 5 th is the first training set and the data in June 6 is the testing set. Similarly, the final data set is from June 5 to June 10 and there are five data sets all together for the observation site. Then, the average of the hybrid model would be calculated as the final forecasting result. In each day, the short-term wind speed data is of 10-minute intervals from 0:00 to 23:50. Electricity price and electrical load data sets are collected from New South Wales (NSW). The scope of data set is from 0:00 to 23:30 during June 1 and June 20 with 30 -minute intervals. Every fifteen days is the training set and the sixteenth day is the testing set. The average of the hybrid model is regarded as the final forecasting result.

3.2. Data Preprocessing. Based on the EEMD method, the original data with white noise is decomposed into seven IMFs, and the sums of the rest of the IMFs are calculated after removing the first two IMFs. Taking the wind speed data set from three observation sites, for example, the curves of processed data are smoother than that of the original data, as shown in Figure 3. Therefore, the establishment and evaluation of the hybrid model should use the data processed by EEMD.

3.3. Evaluation Metrics. Constructing models that could forecast indices in the electrical power system accurately is important; however, what is more important is to set the standard to measure the forecasting performance of the model. The evaluation metrics in this paper would assess the forecasting ability of the model in three aspects, including forecasting accuracy of the single point, the overall forecasting accuracy of multiple points, and the stability of the forecasting.

First, as for the evaluation of forecasting accuracy of the single point, the common and effective way is to calculate the error between forecasting value and actual value, which is defined as absolute percentage error (APE) in this paper:

$$
\mathrm{APE}_{i}=\operatorname{error} \%=\left(\frac{\left|L_{f}-L_{\alpha}\right|}{L_{\alpha}}\right) \times 100 .
$$

Second, the mean absolute percentage error (MAPE), the mean square error (MSE), and the mean absolute error (MAE) are applied to evaluate the forecasting accuracy when comparing the hybrid model with other methods. It is proved that these three indexes could measure the overall forecasting accuracy of the model well [35]. MAE and MSE are mainly applied to evaluate the deviation between the forecasting value and actual value and the former one is more sensitive to the error than the latter one. Their equations are listed below:

$$
\begin{aligned}
\mathrm{MAPE} & =\text { average error\% } \%\left(\frac{1}{n}\right) \sum_{i=1}^{n} \mathrm{APE}_{i}, \\
\mathrm{MAE} & =\frac{1}{n} \sum_{i=1}^{n}\left|e_{t}\right|=\frac{1}{n} \sum_{i=1}^{n}\left|L_{f}-L_{\alpha}\right|, \\
\mathrm{MSE} & =\frac{1}{n} \sum_{i=1}^{n} e_{t}^{2}=\frac{1}{n} \sum_{i=1}^{n}\left(L_{f}-L_{\alpha}\right)^{2},
\end{aligned}
$$

where $e_{t}=L_{f}-L_{\alpha}, n$ is the sample size, $L_{f}$ represents the actual value in time $t$, and $L_{\alpha}$ refers to the forecasting value in time $t$. 


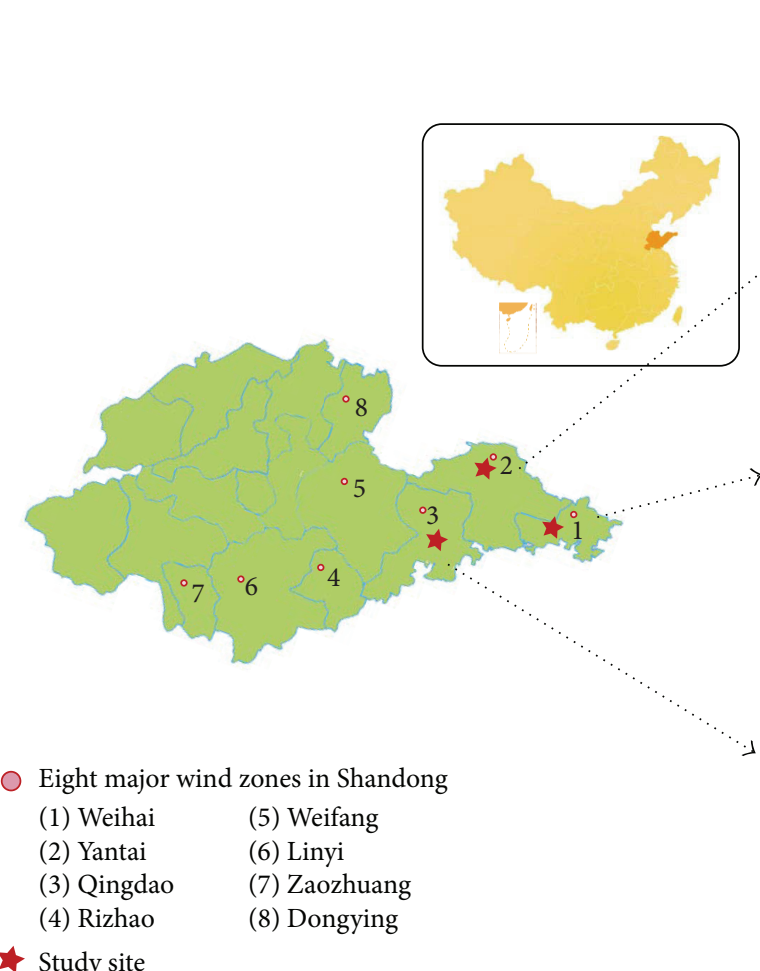

(a)
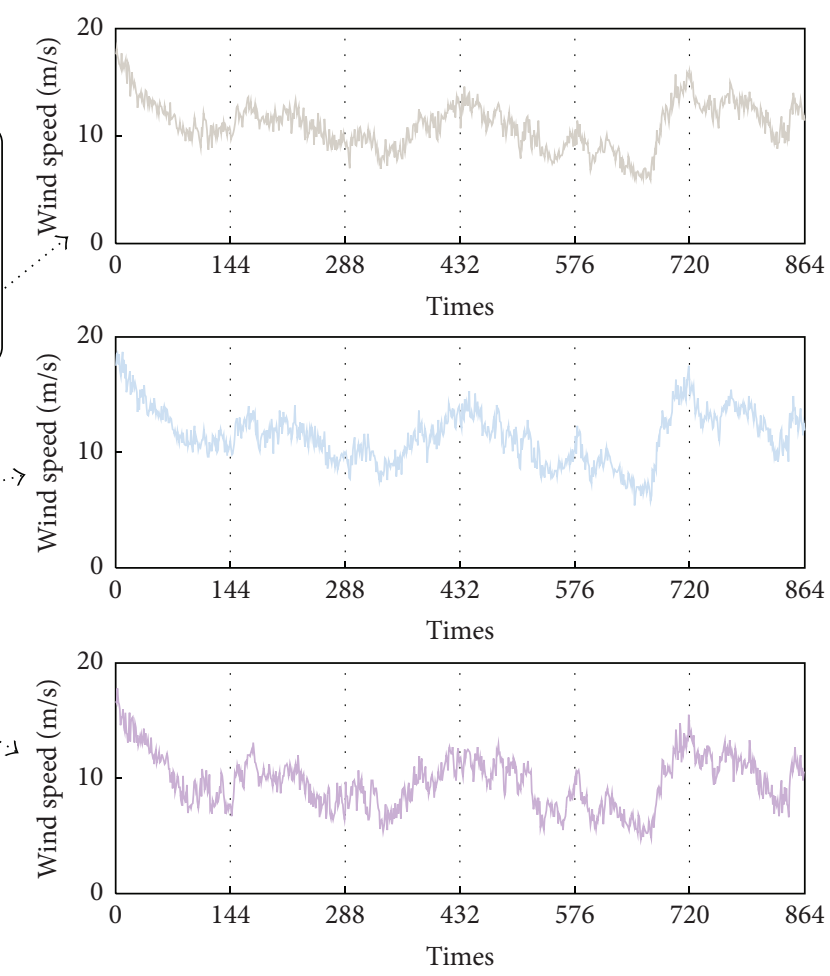

(b)

Statistical measures of original wind speed series

\begin{tabular}{lcccc}
\hline Area & Mean $(\mathrm{m} / \mathrm{s})$ & Standard deviation $(\mathrm{m} / \mathrm{s})$ & Min $(\mathrm{m} / \mathrm{s})$ & $\operatorname{Max}(\mathrm{m} / \mathrm{s})$ \\
\hline Observation site 1 & 11.00 & 2.15 & 5.90 & 18.10 \\
Observation site 2 & 11.44 & 2.29 & 5.30 & 18.70 \\
Observation site 3 & 9.68 & 2.27 & 4.70 & 17.80 \\
\hline
\end{tabular}

(c)

Figure 2: Description of three observation sites in Shandong Peninsula of China.

There is a type of criteria for MAPE to measure the forecasting quality, which is listed in Table 1.

In addition, this paper employs maximum percentage error (MPE) to evaluate the maximum forecasting error, which could measure the stability of the forecasting:

$$
\operatorname{MPE}=\operatorname{Max}\left(\frac{\left|L_{f}-L_{\alpha}\right|}{L_{\alpha}}\right) .
$$

3.4. Experimental Setup. In this paper, we design three experiments to verify the effectiveness of the proposed model, which are Experiment I, Experiment II, and Experiment III. Experiment I compares the proposed hybrid model with its basic form, including MFA-BP and E-BP. Experiment II contains the contrast between E-MFA-BP and traditional well-known forecasting models of time series data. Experiment III compares the optimization algorithm of MFA with other intelligent optimization algorithms when optimizing weight and threshold of BPNN. The data sets applied to the experiments are short-term wind speed time series data,
TABLE 1: Criterion of MAPE [6].

\begin{tabular}{lc}
\hline MAPE (\%) & Forecasting ability \\
\hline$<10$ & Excellent \\
$10-20$ & Good \\
$20-50$ & Reasonable \\
$>50$ & Incorrect \\
\hline
\end{tabular}

electrical load data, and electricity price data. The detailed selection of data has been discussed in Section 3.1.

Experiment $I$ is aimed at comparing E-MFA-BP with MFA-BP and E-BP. Through comparing E-MFA-BP with MFA-BP, the effect of data denoising of EEMD could be verified. Similarly, the comparison between E-MFA-BP and E-BP could prove the optimization effect of MFA.

The purpose of Experiment II is to compare the hybrid model proposed in this paper with five other well-known time series forecasting methods, including AR, ARIMA, BPNN, GM, and SVM. The first two methods belong to traditional statistical models and the latter three models are 

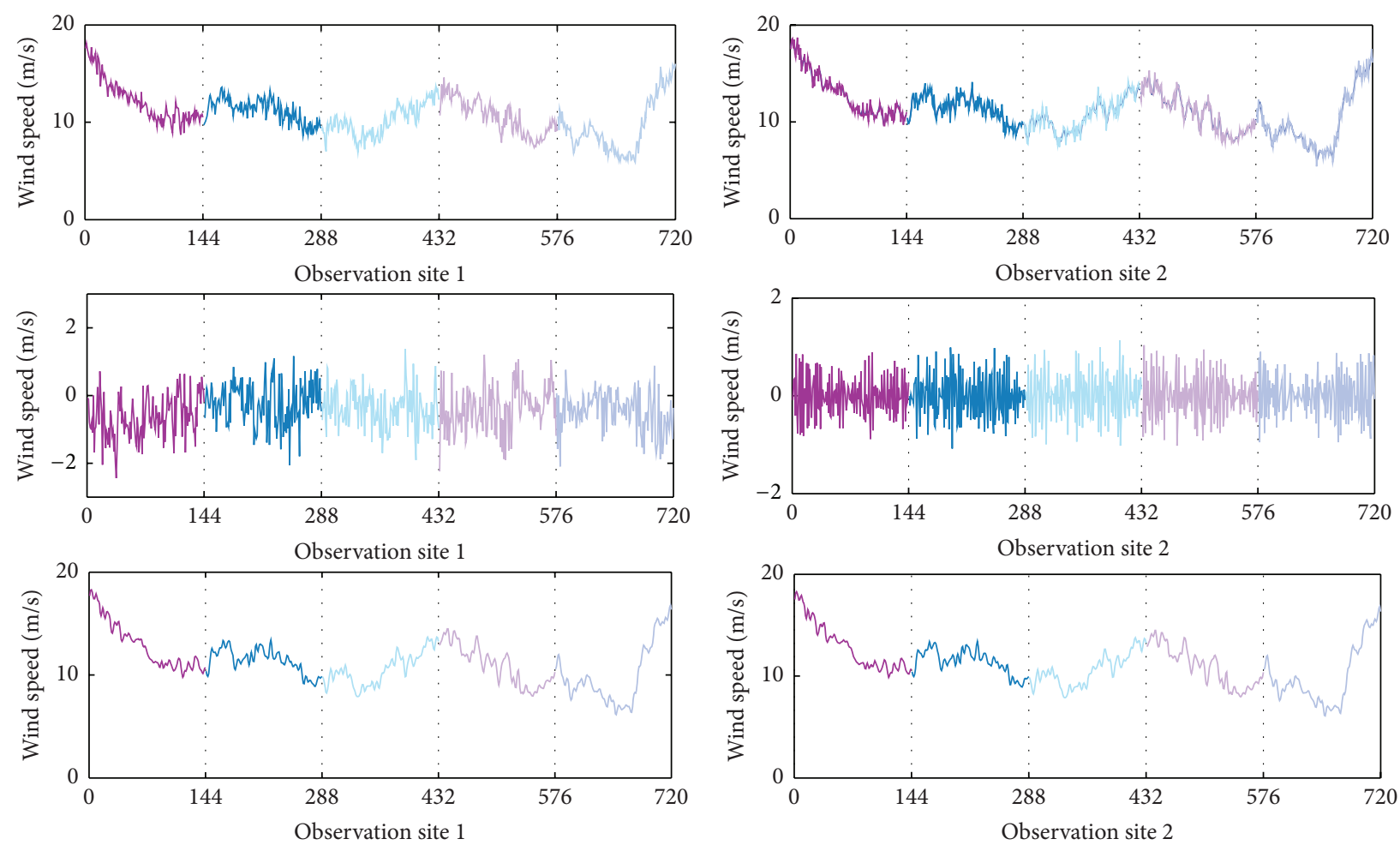

(a) Original data, noise, and data processed by EEMD at observation site

(b) Original data, noise, and data processed by EEMD at observation site 2
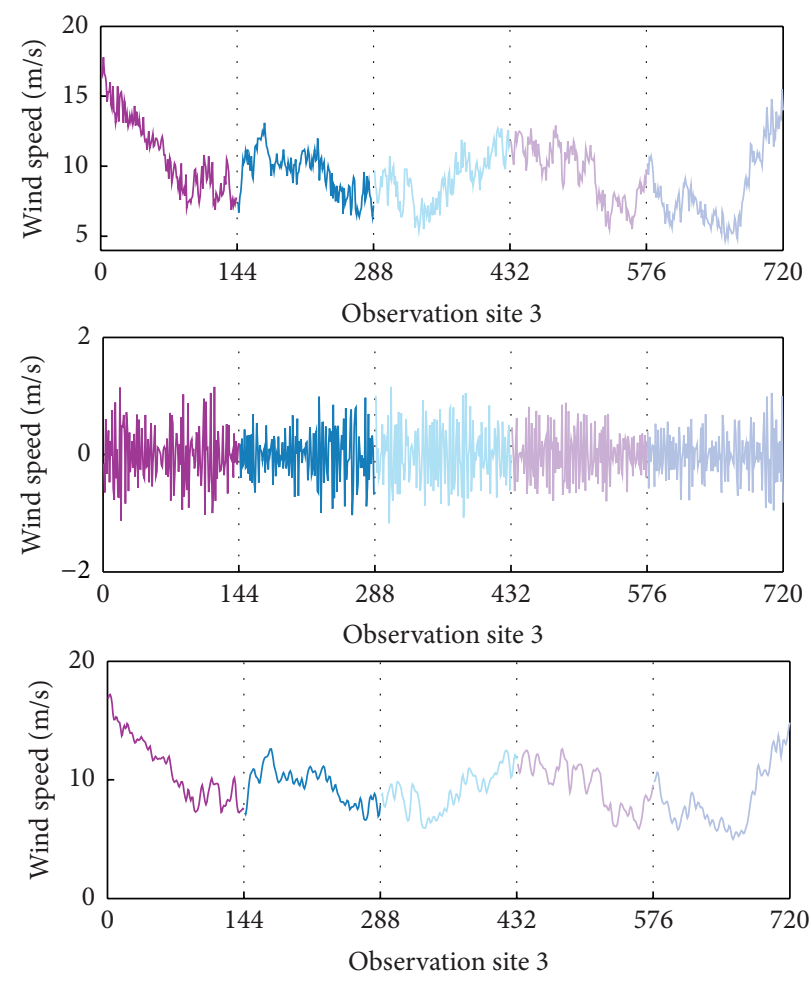

(c) Original data, noise, and data processed by EEMD at observation site 3

FIGURE 3: Comparison between original data and data processed by EEMD for short-term wind speed data sets collected from three observation sites. 
the artificial intelligent ways. Through the comparison, the effectiveness of the model could be verified. Besides, the difference between statistical methods and network ways could be figured out as well from the comparison. The data set is large enough to support the application of both statistical ways and network methods.

Experiment III is designed to contrast different optimization algorithms with MFA used in this paper in the aspect of effect and time of the optimization. It is a significant step to optimize the weight and threshold of BPNN by applying optimization algorithm, because the optimization could help BPNN achieve a better forecasting accuracy. The current popular optimization algorithms include artificial fish swarm algorithm (AFSA), generic algorithm (GA), Cuckoo algorithm (CA), ant colony algorithm (ACA), simulated annealing (SA), and particle swarm optimization (PSO). When carrying out the comparison, the other conditions of EEMD and BPNN would stay unchanged.

For the wind speed data sequence, the first 720 items of data are regarded as the training sample and the latter 144 items of data are applied to test the forecasting accuracy. For electrical load data and electricity price data, the first 720 items of data are the training sample and the rest 48 items of data are the testing data set. The detailed data selection scheme of short-term wind speed is described in Figure 4. The study of the change of parameter in the optimization algorithms is not included in the research scope in this paper; therefore, as for the settings of parameters, the literature would be referred to. Firstly, for BPNN, according to the actual situation, the number of input nodes is 3 , output nodes is 1 , and hidden nodes is 6. Iteration is 100 , learning rate is 0.1 , and goal of accuracy is 0.0001 . The output result of BPNN is not stable, so, for each output, this paper would take the average of the output for 10 times as the final results. Then, for EEMD, the first two decomposed layers are removed. The population of MFA is set to 100 .

In our experiments, the input node is 3 and output node is 1 ; then we test BPNN with different hidden node, including 5 , $6,7,8,9$, and 10 . The difference among different hidden nodes of three data sets is shown in Table 2. The results of MAPE indicate that when the hidden node is 6 , the forecasting quality of the hybrid model is the best; however, the number of hidden node is not a key factor that has a significant influence on the forecasting performance.

3.5. Experiment I. Table 3 demonstrates the forecasting results of the three models, including MFA-BP, E-BP, and EMFA-BP. It is obvious that the hybrid model proposed in this paper achieves the best performance compared with the other two models in the aspect of APE, MAPE, MAE, MSE, and MPE.

In detail, first of all, for the sequence of short-term wind speed, E-MFA-BP has the lowest APE at time of 8:00, 12:00, 16:00, and 20:00 with APEE of 3.90\%, 2.11\%, 4.42\%, and $3.36 \%$, respectively. E-BP performs the best at time of 0:00 and 4:00 with APE of $1.28 \%$ and $2.08 \%$.

For electrical load time series, at the time of 8:00 and 20:00, E-BP has the lowest APE and at other points the proposed hybrid model outperforms the other two models. MAPE
TABLE 2: Comparison of E-MFA-BP with different numbers of hidden nodes.

\begin{tabular}{lccc}
\hline $\begin{array}{l}\text { Number of } \\
\text { hidden nodes }\end{array}$ & Wind speed & $\begin{array}{r}\text { MAPE (\%) } \\
\text { Electrical load }\end{array}$ & Electricity price \\
\hline 3 & 3.95 & 1.32 & 5.50 \\
4 & 3.89 & 1.33 & 5.49 \\
5 & 3.86 & 1.31 & 5.48 \\
6 & 3.86 & 1.23 & 5.56 \\
7 & 3.87 & 1.26 & 5.43 \\
8 & 3.93 & 1.27 & 5.50 \\
9 & 3.98 & 1.25 & 5.48 \\
10 & 3.90 & 1.29 & 5.48 \\
11 & 3.88 & 1.30 & 5.47 \\
12 & 3.92 & 1.27 & 5.44 \\
\hline
\end{tabular}

of E-MFA-BP is $1.23 \%$ with decrease of $1.74 \%$ and $0.25 \%$ compared with MFA-BP and E-BP. The changes of MAE and MSE are not obvious. As for MPE, the hybrid model also maintains the smallest value.

For electricity price time series, E-BP also has better APE than MFA-BP at two points. As for MAPE, although MFABP has higher value compared with E-BP, the hybrid model really achieves a better MPE, which indicates that the model has better forecasting stability. At the time of 4:00, MFABP has larger APE owing to the lower electricity price at that time. From the results it could be found out that the forecasting value of hybrid model put forward is more stable and it improves the forecasting accuracy by nearly $2.79 \%$. Besides, the lower MAE and MSE signify smaller difference between forecasted value and actual value.

EEMD plays an important role in the hybrid model, because the effect of its denoising is evident. For wind speed and electricity price time series, MAPE could improve approximately $3 \%$. For electrical load time series, the actual value of data is big; therefore, the error becomes small relatively. The forecasting accuracy is high; the contribution of EEMD in MAPE is nearly $1.64 \%$.

3.6. Experiment II. Table 4 shows the comparison result between E-MFA-BP and other traditional time series forecasting models, from which it could be found that E-MFABP outperforms all the other models except support vector mechanism (SVM) in MAE of wind speed and electrical load time series and MAPE of electricity price time series.

For wind speed time series, the proposed hybrid model achieves the best performance in MAPE and MSE. E-MFA$\mathrm{BP}$ has the best forecasting accuracy of the multiple points. In comparison, $\mathrm{AR}$ has the worst forecasting performance, because AR is more suitable for forecasting the data with linear and stable trend. There is a large improvement between the proposed hybrid model and AR, ARIMA, and basic BPNN.

For electricity load time series, MAPE of E-MFA-BP is $1.23 \%$ with $1.99 \%$ decrease compared with BPNN. The forecasting result of the hybrid model and SVM is close, 


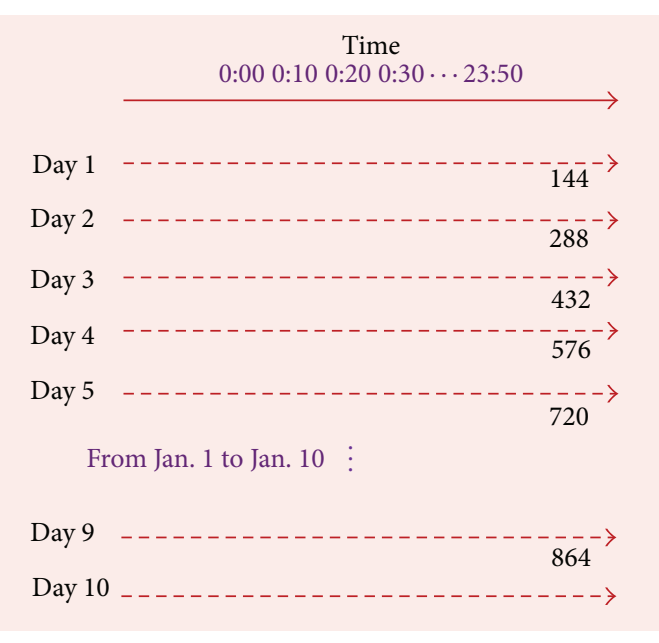

(a) Original data is from Jan. 1 to Jan. 10

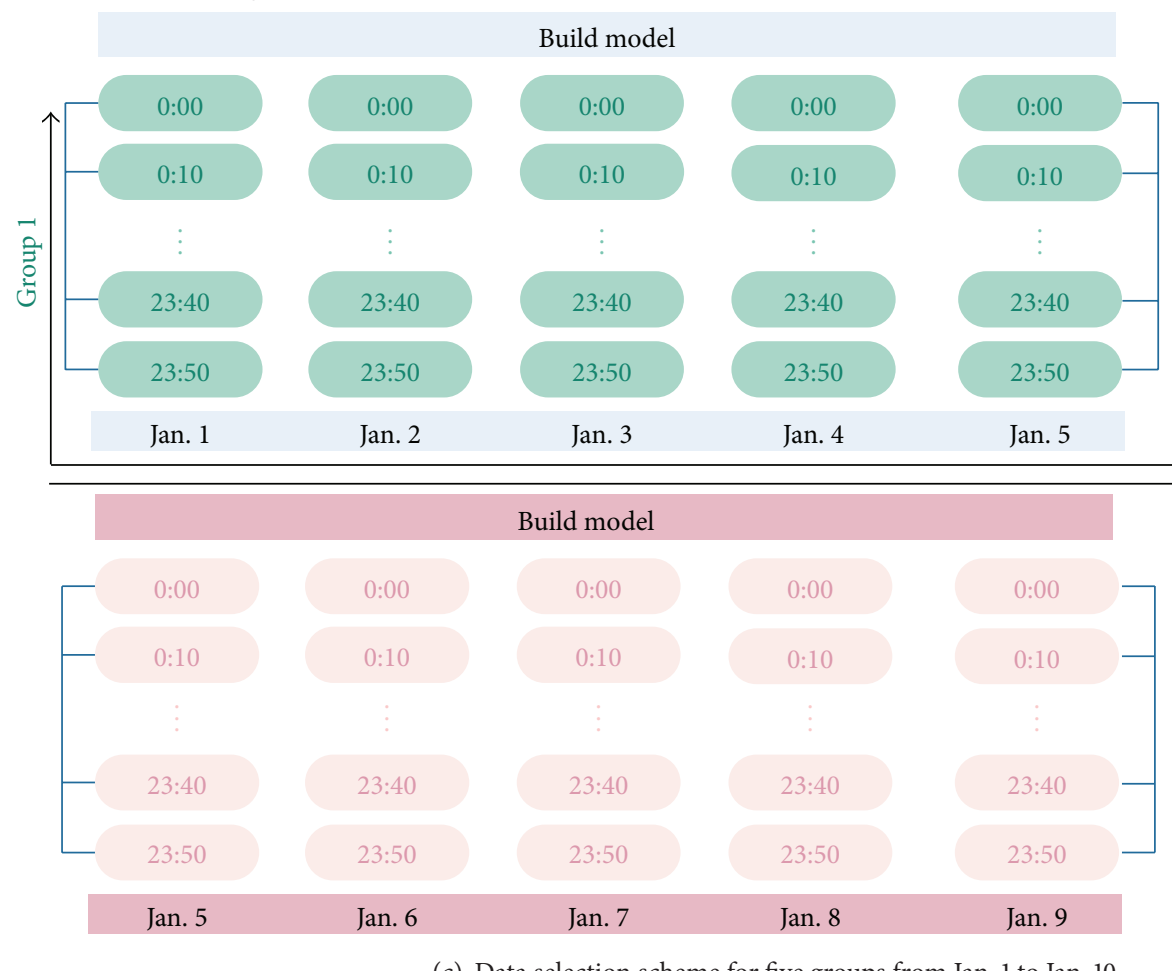

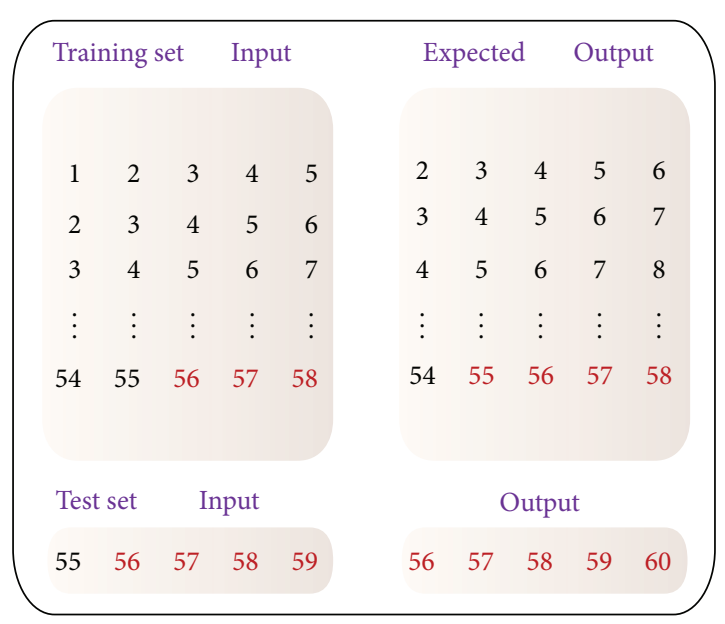

(b) Data structure of the observation sites
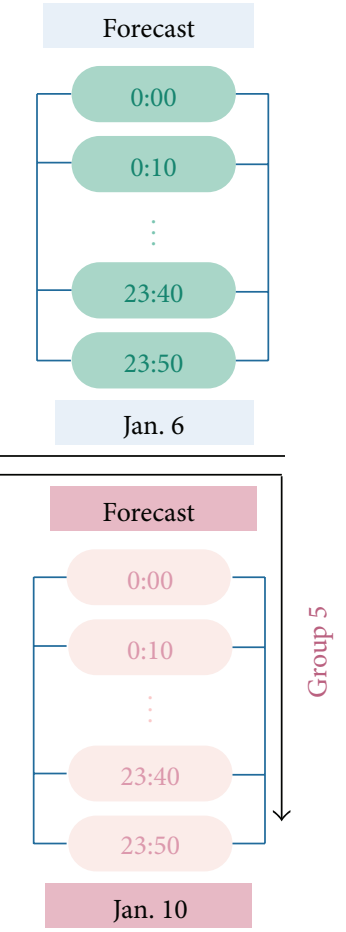

Jan. 10

(c) Data selection scheme for five groups from Jan. 1 to Jan. 10

FIgURE 4: The original data, data structure of the observation sites, and data selection scheme of short-term wind speed.

because the forecasting accuracy of electrical load time series is high owing to the large value of the data. MAE of SVM is 92.781, which is the smallest among all the models.

Although MAPE of electricity price time series of SVM is lower than E-MFA-BP, the hybrid model has better performance in other metrics, especially MSE. In truth, both grey model (GM) and SVM have excellent forecasting accuracy in MAPE, MSE, and MAE. Nevertheless, the ability to forecast the trend of the time series is weaker.

3.7. Experiment III. Table 5 shows the forecasting results by applying different optimization algorithms, including AFSA, FA, GA, CA, ACA, PSO, SA, and MSA. These algorithms are all well-known and common in optimizing parameters in the model. Each single metaheuristic has its own advantages and disadvantages that may be suitable to be applied to optimize the weight and threshold of BPNN. For wind speed time series, MFA outperform all the other methods in all evaluation criteria, although the forecasting stability of FA and ACA is also excellent. Both ACA and MFA have MAPE lower than $7 \%$, which indicates a low error of forecasting. For electrical load time series, the difference among each optimization algorithm is not significantly obvious as well. GA has the best MAPE and MAE and MFA has the best MSE and MPE. MAPEs of all the optimization algorithms are around $3 \%$, which manifests that when forecasting time series 
TABLE 3: The actual and forecasted value and APE obtained from MFA-BP, E-BP, and E-MFA-BP and the value of evaluation criteria.

\begin{tabular}{|c|c|c|c|c|c|c|c|}
\hline \multirow{2}{*}{ Time } & \multirow{2}{*}{ Actual } & \multicolumn{2}{|c|}{ MFA-BP } & \multicolumn{2}{|c|}{ E-BP } & \multicolumn{2}{|c|}{ E-MFA-BP } \\
\hline & & Forecasted & APE (\%) & Forecasted & APE (\%) & Forecasted & $\operatorname{APE}(\%)$ \\
\hline \multicolumn{8}{|c|}{ Short-term wind speed time series } \\
\hline 0:00 & 7.8 & 8.5 & $8.97 \%$ & 7.9 & $1.28 \%$ & 8.1 & $3.85 \%$ \\
\hline 4:00 & 9.6 & 8.8 & $8.33 \%$ & 9.4 & $2.08 \%$ & 9.3 & $3.12 \%$ \\
\hline 8:00 & 7.7 & 7 & $9.09 \%$ & 7.3 & $5.19 \%$ & 7.4 & $3.90 \%$ \\
\hline $12: 00$ & 9.5 & 10.2 & $7.37 \%$ & 9.9 & $4.21 \%$ & 9.3 & $2.11 \%$ \\
\hline $16: 00$ & 11.3 & 10.3 & $8.85 \%$ & 12 & $6.19 \%$ & 11.8 & $4.42 \%$ \\
\hline 20:00 & 11.9 & 10.9 & $8.40 \%$ & 11.4 & $4.20 \%$ & 11.5 & $3.36 \%$ \\
\hline \multicolumn{2}{|c|}{ MAPE (\%) } & \multicolumn{2}{|c|}{$6.62 \%$} & \multicolumn{2}{|c|}{$4.31 \%$} & \multicolumn{2}{|c|}{$3.86 \%$} \\
\hline \multicolumn{2}{|c|}{ MAE } & \multicolumn{2}{|c|}{0.520} & \multicolumn{2}{|c|}{0.497} & \multicolumn{2}{|c|}{0.468} \\
\hline \multicolumn{2}{|c|}{ MSE } & \multicolumn{2}{|c|}{0.472} & \multicolumn{2}{|c|}{0.392} & \multicolumn{2}{|c|}{0.389} \\
\hline \multicolumn{2}{|c|}{ MPE } & \multicolumn{2}{|c|}{$11.03 \%$} & \multicolumn{2}{|c|}{$6.20 \%$} & \multicolumn{2}{|c|}{$4.51 \%$} \\
\hline \multicolumn{8}{|c|}{ Electrical load time series } \\
\hline $0: 00$ & 9185.1 & 9441.2 & $2.79 \%$ & 9315.2 & $1.42 \%$ & 9289.7 & $1.14 \%$ \\
\hline 4:00 & 7144.9 & 7338.2 & $2.71 \%$ & 7280.6 & $1.90 \%$ & 7253.0 & $1.51 \%$ \\
\hline 8:00 & 10356.9 & 10579.2 & $2.15 \%$ & 10488.6 & $1.27 \%$ & 10502.5 & $1.41 \%$ \\
\hline 12:00 & 9950.7 & 10273.2 & $3.24 \%$ & 10215.3 & $2.66 \%$ & 10111.4 & $1.61 \%$ \\
\hline $16: 00$ & 9708.8 & 9962.4 & $2.61 \%$ & 9871.5 & $1.67 \%$ & 9797.5 & $0.91 \%$ \\
\hline 20:00 & 11073.1 & 10724.1 & $3.15 \%$ & 10835.6 & $2.15 \%$ & 10801.1 & $2.46 \%$ \\
\hline \multicolumn{2}{|c|}{ MAPE (\%) } & \multicolumn{2}{|c|}{$2.97 \%$} & \multicolumn{2}{|c|}{$1.48 \%$} & \multicolumn{2}{|c|}{$1.23 \%$} \\
\hline \multicolumn{2}{|c|}{ MAE } & & & & & & \\
\hline & & & & & & & \\
\hline & & & & & & & \\
\hline & & & Elect & ce time series & & & \\
\hline $0: 00$ & 26.6 & 29.28 & $9.91 \%$ & 28.25 & $6.04 \%$ & 27.98 & $5.03 \%$ \\
\hline 4:00 & 18.8 & 20.68 & $10.00 \%$ & 19.95 & $6.12 \%$ & 19.83 & $5.48 \%$ \\
\hline 8:00 & 33.7 & 30.82 & $8.60 \%$ & 31.73 & $5.90 \%$ & 32.01 & $5.07 \%$ \\
\hline $12: 00$ & 28.9 & 31.33 & $8.22 \%$ & 30.96 & $6.94 \%$ & 30.45 & $5.18 \%$ \\
\hline $16: 00$ & 29.9 & 32.47 & $8.49 \%$ & 31.63 & $5.68 \%$ & 31.63 & $5.68 \%$ \\
\hline 20:00 & 34.4 & 31.84 & $7.55 \%$ & 33.06 & $4.01 \%$ & 32.65 & $5.20 \%$ \\
\hline & $\%)$ & & & & & & \\
\hline & & & & & & & \\
\hline & & & & & & & \\
\hline & & & & & & & \\
\hline
\end{tabular}

TABLE 4: Comparison and contrast of E-MFA-BP proposed with other traditional time series forecasting models.

\begin{tabular}{|c|c|c|c|c|c|c|}
\hline & $\mathrm{AR}$ & ARIMA & BPNN & GM & SVM & E-MFA-BP \\
\hline \multicolumn{7}{|c|}{ Short-term wind speed time series } \\
\hline MAPE (\%) & $9.83 \%$ & $8.25 \%$ & $7.38 \%$ & $4.82 \%$ & $4.03 \%$ & $3.86 \%$ \\
\hline MAE & 0.779 & 0.782 & 0.631 & 0.595 & 0.367 & 0.468 \\
\hline MSE & 0.734 & 0.644 & 0.568 & 0.428 & 0.422 & 0.389 \\
\hline \multicolumn{7}{|c|}{ Electrical load time series } \\
\hline MAPE (\%) & $4.87 \%$ & $2.85 \%$ & $3.22 \%$ & $2.51 \%$ & $1.33 \%$ & $1.23 \%$ \\
\hline MAE & 316.782 & 258.966 & 230.090 & 101.438 & 92.781 & 95.919 \\
\hline $\operatorname{MSE}\left(10^{4}\right)$ & 3.013 & 2.665 & 2.874 & 1.593 & 1.688 & 1.488 \\
\hline \multicolumn{7}{|c|}{ Electricity price time series } \\
\hline MAPE (\%) & $8.62 \%$ & $7.33 \%$ & $6.37 \%$ & $5.36 \%$ & $5.02 \%$ & $5.56 \%$ \\
\hline MAE & 5.007 & 4.283 & 3.426 & 4.225 & 3.189 & 2.505 \\
\hline MSE & 139.428 & 100.690 & 58.259 & 89.517 & 37.621 & 11.788 \\
\hline
\end{tabular}


TABLE 5: Comparison of other metaheuristics with MFA in the aspect of MAPE, MAE, MSE, and MPE.

\begin{tabular}{|c|c|c|c|c|c|c|c|c|}
\hline & AFSA & FA & GA & $\mathrm{CA}$ & ACA & PSO & SA & MFA \\
\hline \multicolumn{9}{|c|}{ Short-term wind speed time series } \\
\hline MAPE (\%) & $7.21 \%$ & $7.35 \%$ & $7.08 \%$ & $7.53 \%$ & $6.92 \%$ & $7.83 \%$ & $7.16 \%$ & $6.62 \%$ \\
\hline MAE & 0.739 & 0.881 & 0.926 & 0.765 & 0.538 & 0.559 & 0.619 & 0.520 \\
\hline MSE & 0.662 & 0.619 & 0.538 & 0.482 & 0.561 & 0.722 & 0.491 & 0.472 \\
\hline MPE & $8.65 \%$ & $7.58 \%$ & $7.74 \%$ & $9.62 \%$ & $7.35 \%$ & $8.56 \%$ & $9.78 \%$ & $6.73 \%$ \\
\hline \multicolumn{9}{|c|}{ Electrical load time series } \\
\hline MAPE (\%) & $3.10 \%$ & $3.28 \%$ & $2.88 \%$ & $3.17 \%$ & $3.24 \%$ & $2.98 \%$ & $3.23 \%$ & $2.97 \%$ \\
\hline MAE & 118.623 & 115.228 & 101.354 & 128.095 & 137.342 & 117.908 & 123.659 & 103.090 \\
\hline $\operatorname{MSE}\left(10^{4}\right)$ & 2.983 & 2.991 & 2.315 & 2.442 & 2.187 & 2.099 & 2.679 & 2.014 \\
\hline MPE & $3.42 \%$ & $3.76 \%$ & $3.29 \%$ & $3.66 \%$ & $4.01 \%$ & $3.59 \%$ & $3.87 \%$ & $3.33 \%$ \\
\hline \multicolumn{9}{|c|}{ Electricity price time series } \\
\hline MAPE (\%) & $9.87 \%$ & $9.63 \%$ & $9.42 \%$ & $8.81 \%$ & $9.28 \%$ & $9.10 \%$ & $8.90 \%$ & $8.75 \%$ \\
\hline MAE & 4.218 & 4.442 & 3.917 & 4.258 & 4.937 & 5.018 & 4.005 & 3.894 \\
\hline MSE & 100.235 & 78.096 & 121.305 & 100.266 & 76.302 & 66.675 & 98.376 & 57.025 \\
\hline MPE & $11.26 \%$ & $10.37 \%$ & $12.83 \%$ & $9.90 \%$ & $11.24 \%$ & $10.76 \%$ & $10.45 \%$ & $9.55 \%$ \\
\hline
\end{tabular}

data with high forecasting accuracy, the role the optimization algorithm plays is not decisive. Therefore, we can choose one based on their rate of convergence. For the last time series of electricity price, CA, SA, and MFA are superior to AFSA, FA, GA, ACA, SA, and PSO, because they have MAPE lower than $9 \%$. As for MPE, there is no question that the proposed MFA has the best performance. However, there are only two algorithms, including GA and CA, having MPE lower than $7 \%$.

\section{Discussion}

There is a large variety of forecasting models that could be utilized in time series data, including statistical models and artificial intelligent neural networks. As discussed above, the accurate forecasting of indices in the electrical power system is pivotal; thus, to choose the best forecasting model seems extraordinarily important.

4.1. Statistical Models. The statistical models are popular and common in the forecasting; therefore, this paper chooses two models, which are AR and ARIMA, to carry out the comparison with the hybrid model proposed. According to the experimental results, it could be known that ARIMA has better forecasting performance than AR and this is because ARIMA is a generalization form of ARMA. That is to say that ARIMA would be more effective in the prediction of time series data. The largest weakness of statistical models is that a fairly linear trend and small fluctuations are required. If the data sequence is full of noise and has a high degree of fluctuation, the statistical models would become invalid, which means that the forecasting errors are so big that they cannot be accepted in the practical application. ARIMA has an extra parameter named differencing degree that represents the number of nonseasonal differences to fine tune the models to be more accurate based on ARMA [6]. Therefore, ARIMA contains a process of smoothing the original data compared with AR which is the most basic form of statistical models. The three data sets selected in the experiments all include irregular information and high fluctuation. So the application of statistical models is weak.

4.2. Neural Networks. Neural networks applied as nonlinear forecasting models have obtained huge popularity and success in time series forecasting and this is because it is proved that the neural networks outperform the other statistical models. In our experiment, the results agree with the points of view above.

From Experiment II, it could be found that neural networks including BPNN and the hybrid model gain better results than AR and ARIMA in the aspect of MAPE, MAE, MSE, and MPE, although, for electoral load time series, MAPE of BPNN is larger than ARIMA. However, for the other two data sets of wind speed and electricity price data sets, all the neural networks outperform statistical models. In addition, as for the value of MPE, the metrics of evaluating the stability of forecasting, neural networks also have better performance compared with traditional statistical models, because the three data sets in the electrical power system all obey nonlinear distribution and contain irregular information. The neural network could deal with such problems commendably through training the network continuously. In comparison, the forecasting quality of single BPNN is worse than the other models, including GM, SVM, and the proposed hybrid model E-MFA-BP. Due to the instability of BPNN, our experiment calculates the average value of forecasting for 10 times in order to get over the forecasting error owing to the stable results. For wind speed data set, the train-to-verify ratio is $5: 1$ and for electrical load and electricity price data set, the train-to-verify ratio is $15: 1$. During our experiment, the train-to-verify ratio is also set as $4: 1$ and $3: 1$ for wind speed data set and 12:1 and $9: 1$ for the other two data sets. The experiment results demonstrate that the train-to-verify ratio would not affect the forecasting accuracy obviously. 


\subsection{The Proposed Hybrid Model}

4.3.1. Effect of Denoising. As discussed above, EEMD is an effective method to eliminate the noise in the original data set. This paper applies EEMD to carry out the denoising and the results prove that the effect of EEMD is so evident that the forecasting accuracy gets improved to a large degree after adding EEMD. From Experiment $I$, it is obvious that, for wind speed data of three observation sites, MAPEs increase by nearly $2 \%$. For electrical load time series and electricity price time series, MAPE rises by about $1.7 \%$ and $2.6 \%$, respectively. As for the other indices, including MAE and MSE, they are improved as well. The experiment proves the effectiveness of EEMD completely. In this paper, we remove the first two columns of output of EEMD. We also study the performance of EEMD when the first three, four, and five columns are removed. The experiment results indicate that when removing the first two output columns, the forecasting result is the closest to the actual values. There is no large difference between removing the first two and three columns.

4.3.2. Effect of Metaheuristics. BPNN optimized by MFA is applied to forecast the time series data denoised by EEMD, including wind speed, electrical load, and electricity price. The single BPNN cannot achieve a high forecasting accuracy because of the randomness and probability mechanism in the network and the adjustment of weight and threshold of BPNN is beneficial in improving rate of convergence and stability and reducing the forecasting errors. Weight and threshold are two values that could affect the overall forecasting accuracy of BPNN, because through adjusting these two values the network of BPNN could be trained well and then used to carry out the forecasting. From Experiment III, it could be known that MFA is more suitable in the condition that the forecasting accuracy of the hybrid model is lower relatively. To be specific, MAPE of short-term wind speed and electricity price is about $6.5 \%$ and $8 \%$, respectively, and it can be found obviously from Table 5 that MFA has better performance. However, MAPE of electrical load time series is about $3 \%$, and the optimization effect of MFA is not obvious. The optimization algorithm could improve the forecasting accuracy to a small degree, but it is also significant for the forecasting in the electricity power system.

\section{Conclusion and Further Work}

The electrical power system is a complex system that is composed of many links, including generation, transmission, distribution, and utilization of electricity. This system provides electrical energy for users through converting the primary energy in the nature into electricity. In order to achieve this, the electrical power system also owns relevant information and control system in each section and level so as to ensure that the users obtain safe, economic, and superior electricity. This paper discusses three types of indices in the electrical power system, including short-term wind speed, electrical load, and electricity price. For wind speed data, it is related to the generation of electricity with the increase of focusing on green energy. Then, the electrical load and electricity price concern the balance between demand and supply of electricity. The forecasting of indices plays an important role in managing and controlling the daily operation in the electrical power system. This paper proposes a hybrid model named as E-MFA-BP to carry out the forecasting. The three experiments certify the effectiveness of the hybrid model put forward, because it has better performance compared with other well-known time series forecasting models, and BPNN optimized by other optimization algorithms.

Firstly, EEMD is applied to eliminate the noise in the original wind speed data. The test results indicate that the effect of noise reduction of EEMD is excellent, proving that after utilizing EEMD, the forecasting accuracy is improved to a great degree. Secondly, MFA is used to optimize the weight and threshold values of the net of BPNN. As is well-known to all, the value of the weight and threshold of BPNN could have an influence on forecasting effects. Moreover, BPNN is a common artificial intelligence algorithm for carrying out forecasting; after noise reduction and optimization, BPNN works well in the forecasting of the three indices. Finally, we discuss the comparison of statistical and neural network models and the set of parameters in the hybrid model. In short, the hybrid model proposed in this paper could be applied to forecasting the indices in the electrical power system.

An extension of this work includes keeping the balance of the conflictions between different evaluation metrics by handling the forecasting problems with multiobjectives in fitness function [6].

\section{Abbreviations}

HAP: Hybrid swarm technique

ACO: Ant colony optimization

ARMA: Autoregressive moving model

PSO: Particle swarm optimization

ARIMA: Autoregressive integrated moving average model

RSVR: Robust support vector regression

GARCH: Generalized autoregressive conditional heteroskedasticity

CSA: Clonal selection principle of artificial immune algorithm

ANN: Artificial neural network

MLP: Multilayer perceptron

ME: $\quad$ Mean error

GA: Genetic algorithm

MSE: Mean square error

FAC: $\quad$ First-order adaptive coefficient

MAE: Mean absolute error

SAC: Second-order adaptive coefficient

BPNN: Back propagation neural network

WTT: Wavelet transform technique

EOF: Empirical orthogonal function

SAM: Seasonal adjustment method

KF: Kalman filter

RBFNN: Radial basis function neural network 
WD: Wavelet decomposition

FEEMD: Fast ensemble empirical mode decomposition

WPD: Wavelet packet decomposition

EEMD: Ensemble empirical mode decomposition

EMD: Empirical mode decomposition

MAPE: Mean absolute percentage error

MFA: Modified firefly algorithm

APE: Absolute percentage error

MPE: Maximum percentage error

AFSA: Artificial fish swarm algorithm

CA: Cuckoo algorithm

SA: $\quad$ Simulated annealing

SVM: Support vector mechanism

GM: $\quad$ Grey model

NSW: New South Wales

IMF: Intrinsic mode function

ARG: Autoregressive gamma

GAR: Gamma autoregressive.

\section{Conflict of Interests}

The authors declare that there is no conflict of interests regarding the publication of this paper.

\section{Acknowledgment}

This work was funded by the Support Plan for Leaders of the First-Class Discipline with Characteristics in Colleges and Universities of Liaoning Province of China under Grant no. XKRC-201403.

\section{References}

[1] Z. Su, J. Wang, H. Lu, and G. Zhao, "A new hybrid model optimized by an intelligent optimization algorithm for wind speed forecasting," Energy Conversion and Management, vol. 85, pp. 443-452, 2014.

[2] E. Erdem and J. Shi, "ARMA based approaches for forecasting the tuple of wind speed and direction," Applied Energy, vol. 88, no. 4, pp. 1405-1414, 2011.

[3] M. Monfared, H. Rastegar, and H. M. Kojabadi, "A new strategy for wind speed forecasting using artificial intelligent methods," Renewable Energy, vol. 34, no. 3, pp. 845-848, 2009.

[4] S. Bivona, G. Bonanno, R. Burlon, D. Gurrera, and C. Leone, "Stochastic models for wind speed forecasting," Energy Conversion and Management, vol. 52, no. 2, pp. 1157-1165, 2011.

[5] W. El-Baz and P. Tzscheutschler, "Short-term smart learning electrical load prediction algorithm for home energy management systems," Applied Energy, vol. 147, pp. 10-19, 2015.

[6] L. Liu, Q. R. Wang, J. Z. Wang, and M. Liu, "A rolling grey model optimized by particle swarm optimization in economic prediction," Computational Intelligence, 2014.

[7] K. He, Y. Xu, Y. Zou, and L. Tang, "Electricity price forecasts using a Curvelet denoising based approach," Physica A: Statistical Mechanics and its Applications, vol. 425, pp. 1-9, 2015.

[8] M. Lipperheide, J. L. Bosch, and J. Kleissl, "Embedded nowcasting method using cloud speed persistence for a photovoltaic power plant," Solar Energy, vol. 112, pp. 232-238, 2015.
[9] R. G. Kavasseri and K. Seetharaman, "Day-ahead wind speed forecasting using f-ARIMA models," Renewable Energy, vol. 34, no. 5, pp. 1388-1393, 2009.

[10] H. Liu, E. Erdem, and J. Shi, "Comprehensive evaluation of ARMA-GARCH(-M) approaches for modeling the mean and volatility of wind speed," Applied Energy, vol. 88, no. 3, pp. 724732, 2011.

[11] M. Caporin and J. Preś, "Modelling and forecasting wind speed intensity for weather risk management," Computational Statistics \& Data Analysis, vol. 56, no. 11, pp. 3459-3476, 2012.

[12] M. Bouzerdoum, A. Mellit, and A. M. Pavan, "A hybrid model (SARIMA-SVM) for short-term power forecasting of a smallscale grid-connected photovoltaic plant," Solar Energy, vol. 98, pp. 226-235, 2013.

[13] W. Xu, R. Gu, Y. Liu, and Y. Dai, "Forecasting energy consumption using a new GM-ARMA model based on HP filter: the case of Guangdong Province of China," Economic Modelling, vol. 45, pp. 127-135, 2015.

[14] E. Cadenas and W. Rivera, "Wind speed forecasting in three different regions of Mexico, using a hybrid ARIMA-ANN model," Renewable Energy, vol. 35, no. 12, pp. 2732-2738, 2010.

[15] X. Q. Nan, Q. Z. Li, D. Q. Qiu, Y. Z. Zhao, and X. X. Guo, "Short-term wind speed syntheses correcting forecasting model and its application," International Journal of Electrical Power and Energy Systems, vol. 49, no. 1, pp. 264-268, 2013.

[16] R. Rahmani, R. Yusof, M. Seyedmahmoudian, and S. Mekhilef, "Hybrid technique of ant colony and particle swarm optimization for short term wind energy forecasting," Journal of Wind Engineering and Industrial Aerodynamics, vol. 123, pp. 163-170, 2013.

[17] J. Geng, M.-W. Li, Z.-H. Dong, and Y.-S. Liao, "Port throughput forecasting by MARS-RSVR with chaotic simulated annealing particle swarm optimization algorithm," Neurocomputing, vol. 147, pp. 239-250, 2015.

[18] H. Liu, H.-Q. Tian, C. Chen, and Y.-F. Li, "An experimental investigation of two Wavelet-MLP hybrid frameworks for wind speed prediction using GA and PSO optimization," International Journal of Electrical Power \& Energy Systems, vol. 52, no. 1, pp. 161-173, 2013.

[19] W. Z. Zhang, J. Wu, J. Z. Wang, W. G. Zhao, and L. Shen, "Performance analysis of four modified approaches for wind speed forecasting," Applied Energy, vol. 99, pp. 324-333, 2012.

[20] W. Y. Zhang, J. J. Wang, J. Z. Wang, Z. B. Zhao, and M. Tian, "Short-term wind speed forecasting based on a hybrid model," Applied Soft Computing Journal, vol. 13, no. 7, pp. 3225-3233, 2013.

[21] J. M. Hu, J. Z. Wang, and K. L. Ma, "A hybrid technique for shortterm wind speed prediction," Energy, vol. 81, no. 1, pp. 563-574, 2015.

[22] J. Wang, S. Qin, Q. Zhou, and H. Jiang, "Medium-term wind speeds forecasting utilizing hybrid models for three different sites in Xinjiang, China," Renewable Energy, vol. 76, pp. 91-101, 2015.

[23] C. Sudheer, S. K. Sohani, D. Kumar et al., "A support vector machine-firefly algorithm based forecasting model to determine malaria transmission," Neurocomputing, vol. 129, pp. 279288, 2014.

[24] Q. Hu, S. Zhang, Z. Xie, J. Mi, and J. Wan, "Noise model based $\nu$-support vector regression with its application to short-term wind speed forecasting," Neural Networks, vol. 57, pp. 1-11, 2014. 
[25] J. Z. Wang, J. M. Hu, K. L. Ma, and Y. X. Zhang, "A self-adaptive hybrid approach for wind speed forecasting," Renewable Energy, vol. 78, pp. 374-385, 2015.

[26] J. J. Fister, M. Perc, S. M. Kamal, and I. Fister, "A review of chaosbased firefly algorithms: perspectives and research challenges," Applied Mathematics and Computation, vol. 252, pp. 155-165, 2015.

[27] B. Krawczyk, "One-class classifier ensemble pruning and weighting with firefly algorithm," Neurocomputing, vol. 150, pp. 490-500, 2015.

[28] S.-U. R. Massan, A. I. Wagan, M. M. Shaikh, and R. Abro, "Wind turbine micrositing by using the firefly algorithm," Applied Soft Computing, vol. 27, pp. 450-456, 2015.

[29] X. S. Yang, "Firefly algorithms for multimodal optimization," in Stochastic Algorithms: Foundations and Applications, vol. 5792 of Lecture Notes in Computer Science, pp. 169-178, Springer, Berlin, Germany, 2009.

[30] S. Gholizadeh, "Performance-based optimum seismic design of steel structures by a modified firefly algorithm and a new neural network," Advances in Engineering Software, vol. 81, pp. 50-65, 2015.

[31] S. J. Kang, Research and improvement of glowworm swarm optimization [M.S. thesis], Guangdong University of Technology, Guangzhou, China, 2013, (Chinese).

[32] A. Tascikaraoglu and M. Uzunoglu, "A review of combined approaches for prediction of short-term wind speed and power," Renewable and Sustainable Energy Reviews, vol. 34, pp. 243-254, 2014.

[33] Z. Guo, J. Zhao, W. Zhang, and J. Wang, "A corrected hybrid approach for wind speed prediction in Hexi Corridor of China," Energy, vol. 36, no. 3, pp. 1668-1679, 2011.

[34] X. F. Zhao, Q. Ba, L. Zhou, W. J. Li, and J. P. Ou, "BP neural network recognition algorithm for scour monitoring of subsea pipelines based on active thermometry," Optik, vol. 125, no. 18, pp. 5426-5431, 2014.

[35] L. Y. Xiao, J. Z. Wang, X. S. Yang, and L. Y. Xiao, "A hybrid model based on data preprocessing for electrical power forecasting," International Journal of Electrical Power \& Energy Systems, vol. 64, pp. 311-327, 2015. 


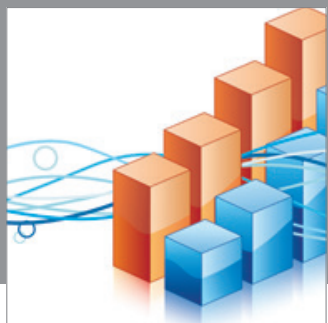

Advances in

Operations Research

mansans

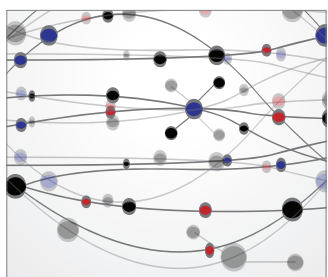

The Scientific World Journal
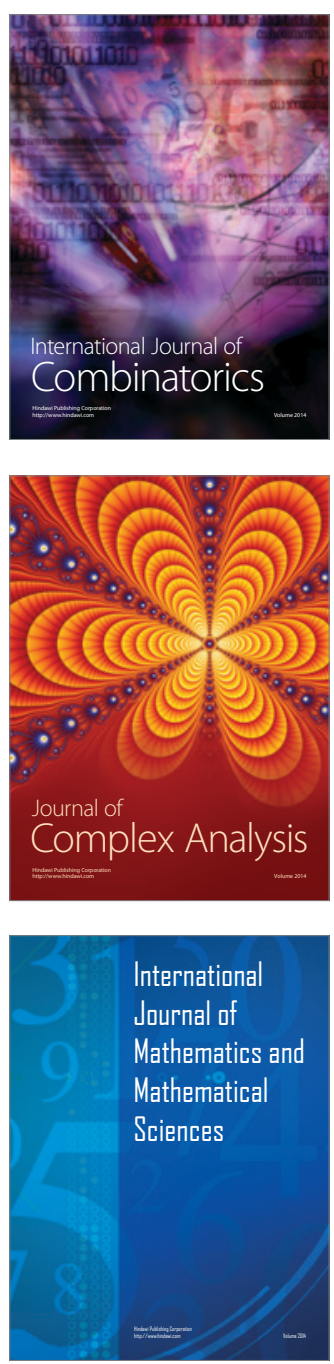
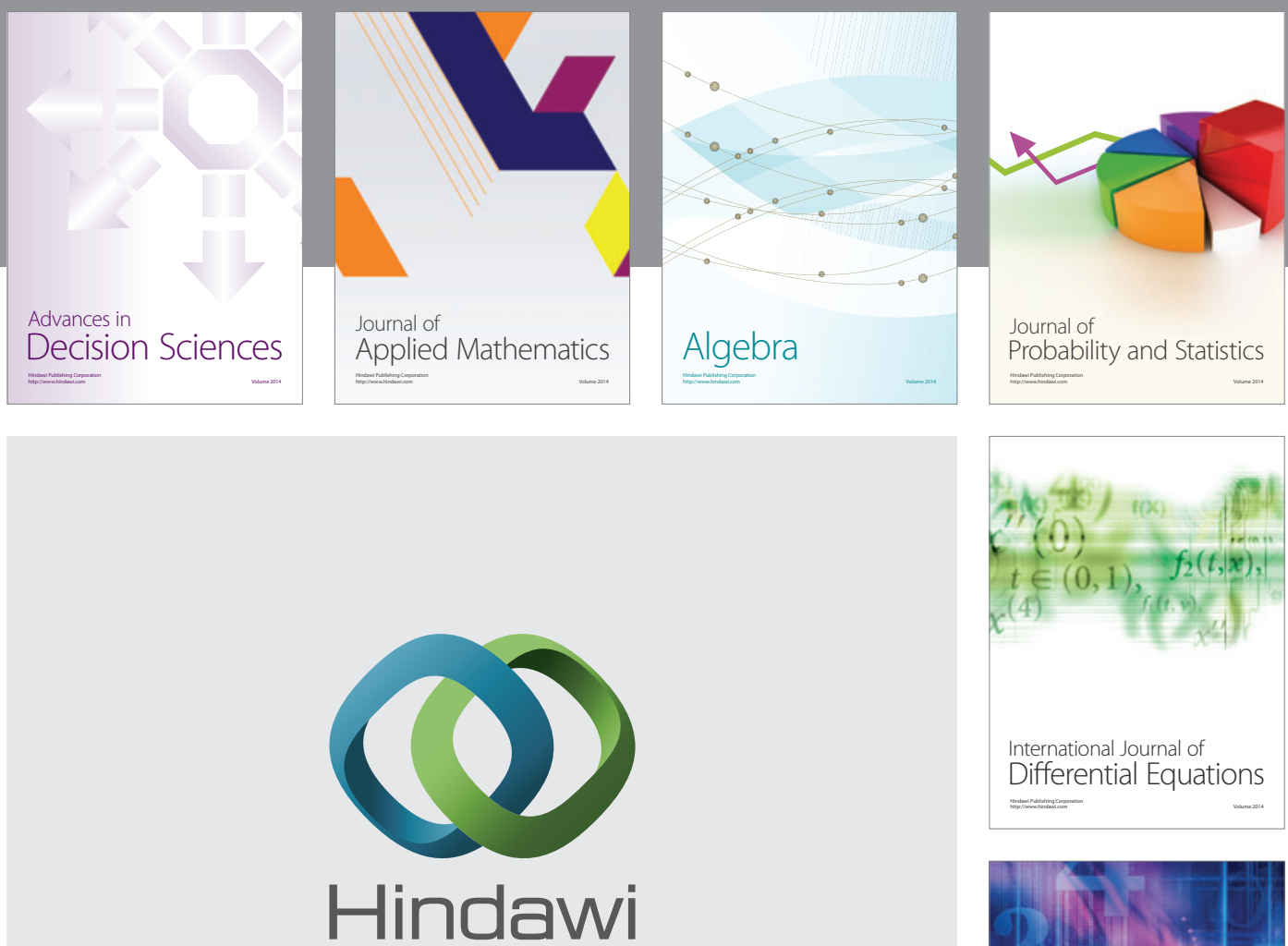

Submit your manuscripts at http://www.hindawi.com
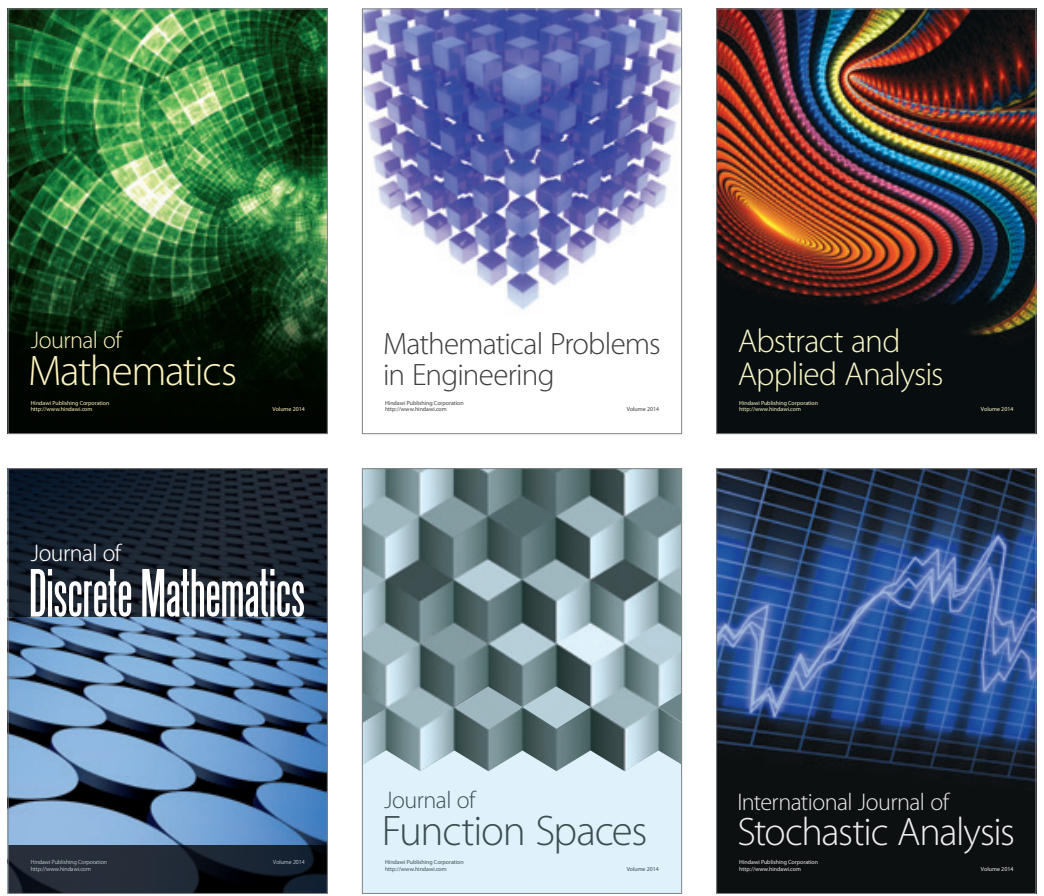

Journal of

Function Spaces

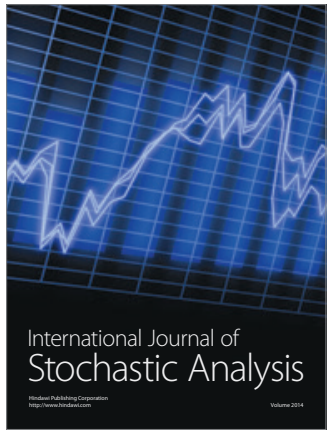

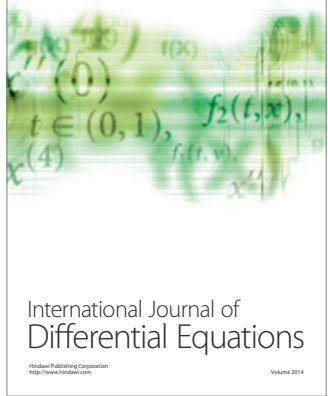
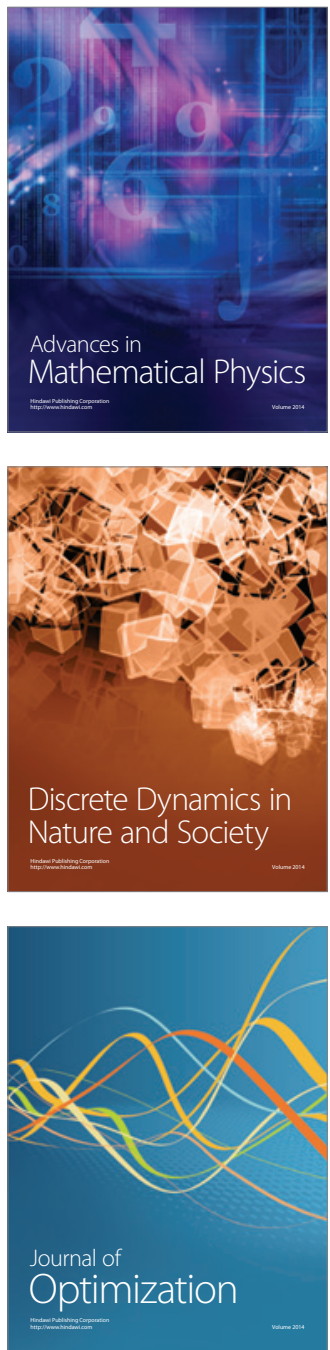